Meta

Journal des traducteurs

Translators' Journal

\title{
Les génératrices
}

\section{Armand Adlerblum}

Volume 23, numéro 3, septembre 1978

URI : https://id.erudit.org/iderudit/001854ar

DOI : https://doi.org/10.7202/001854ar

Aller au sommaire du numéro

Éditeur(s)

Les Presses de l'Université de Montréal

ISSN

0026-0452 (imprimé)

1492-1421 (numérique)

Découvrir la revue

Citer cet article

Adlerblum, A. (1978). Les génératrices. Meta, 23(3), 235-245.

https://doi.org/10.7202/001854ar

Ce document est protégé par la loi sur le droit d'auteur. L’utilisation des services d'Érudit (y compris la reproduction) est assujettie à sa politique d'utilisation que vous pouvez consulter en ligne.

https://apropos.erudit.org/fr/usagers/politique-dutilisation/
Cet article est diffusé et préservé par Érudit.

Érudit est un consortium interuniversitaire sans but lucratif composé de l’Université de Montréal, l'Université Laval et l'Université du Québec à Montréal. Il a pour mission la promotion et la valorisation de la recherche. https://www.erudit.org/fr/ 


\section{LES GÉNÉRATRICES}

Presque toute l'énergie électrique consommée dans le monde s'obtient en déplacant un corps conducteur à l'intérieur des lignes de force d'un aimant. $\mathrm{Ce}$ déplacement induit un courant électrique dans le conducteur. La génératrice la plus simple se compose d'une boucle de fil conducteur les deux pôles d'un aimant pour qu'il coupe son champ magnétique. La rotation de la spire entraine celle des anneaux conducteurs - les bagues collectrices ou collecteurs - dont chacun est reliẹ à l'une des extrémités de la spire. Les bagues sont frottées par des pièces conductrices de métal ou de graphite, les balais, qui transmettent le courant engendré au circuit de charge.

L'énergie électrique, electric energy, mais électronicien, electrical engineer. "Electric" s'applique aux propriétés électriques, "electrical" au domaine de l'ẻlectricité. Ingénieur électricien, electrical engineer; moteur électrique, electric motor.

Lignes de force, flux lines. L'ensemble des lignes de force constitue le champ magnétique, magnetic field, de l'aimant.

Générateur et génératrice, generator. Un générateur est un appareil électronique produisant des signaux. Ex.: générateur de tension continue, de tension sinusoîdale basse fréquence (B.F.) et haute fréquence (H.F.), d'impulsion, etc. Une génératrice est une machine tournante qui convertit l'énergie mécanique en énergie électrique.

Bague collectrice: «Il semble bien que l'anglais ait tendance à employer collector ring, plus utilisé aux Etats-Unis plutôt que slip ring, précédemment préféré au Royaume-Uni pour une bague collectrice. " Jean Maillot, la Traduction scientifique et technique. 
Balais, brush. Autres expressions: contact glissant, sliding contact; balais de contact, balais collecteur, balais de prise de courant - collector brush, current collecting brush, sliding brush.

Circuit de charge, load circuit. La charge est le dispositif alimenté par l'énergie électrique transportée par le circuit : lampe, radio, moteur, sonnerie, etc.

La force électromotrice (f.é.m.) induite et le courant du circuit extérieur augmentent proportionnellement au nombre de lignes de force coupées par la spire durant sa rotation. Ce nombre est nul aux positions $A$ et $C\left(0^{\circ}\right.$ et $\left.180^{\circ}\right)$, et maximal en B et en $\mathrm{D}\left(90^{\circ}\right.$ et $\left.270^{\circ}\right)$ (V. schéma). On obtient donc un courant alternatif, puisque la force électromotrice et le courant qu'elle engendre changent de polarités chaque fois que la spire accomplit une rotation de $180^{\circ}$.

Force electromotrice, electromotive force $(\mathrm{emf})$. Force qui se manifeste lorsque deux points n'ont pas la même quantité de charges électriques (différence de potentiel). Cette force provoque le mouvement des électrons.

Induite, induced. Se dit de l'énergie électrique ou magnétique transmise à distance par l'intermédiaire d'un aimant ou d'un courant.

On peut transformer la génératrice de courant alternatif - ou alternateur - en génératrice de courant continu - ou dynamo. Il suffit pour cela de remplacer les bagues collectrices par un commutateur formé d'un cylindre fendu dont les deux segments sont chacun relié à l'une des bornes de l'armature, et sont isolés l'un de l'autre du point de vue électrique.

Commutateur: J. Maillot écrit, dans la Traduction scientifique et technique: a... commutator n'est pas un commutateur, pour lequel l'anglais emploie des termes dérivés de switch... mais le collecteur d'une machine tournante. "On trouve cependant commutator employé dans ce dernier sens (Cf. Harry Mileaf, Electricity six, Hayden Book Company). Il s'agit en effet d'une commutation, c'est-àdire d'un changement de contact qui renverse le sens du courant. Autres expressions: commutateur rotatif, collecteur, bague collectrice et redresseur tournant. Celle-ci rappelle que la dynamo n'engendre pas directement le courant continu, mais redresse ("rectifies") la partie négative du courant alternatif.

Segment, demi-coquille, ou lame, segment, conductor, ou conducting member.

Armature, partie tournante d'un générateur. Il s'agit ici d'une armature à spire unique. Dans la pratique, l'armature est une bobine («coil») comprenant un grand nombre de spires ( (urns») afin d'obtenir de hautes tensions. On l'appelle aussi bobine d'armature, bobine d'induit ou induit.

Isolés... du point de vue électrique. Ne pas dire électriquement isolés, car l'électricité ne sert pas à isoler. On veut dire ici "électriquement parlant".

L'un des balais est toujours relié au côté descendant de la boucle, qui fait croître la tension, et l'autre au côté ascendant, qui la fait décroître. Ainsi, l'un des balais est toujours positif, et l'autre toujours négatif, contrairement aux balais de l'alternateur, qui changent de polarités après chaque rotation de $180^{\circ}$. Il en résulte un courant de charge qui se dirige toujours dans le même sens, mais qui varie, pour chaque révolution de la spire, de zéro à une valeur maximalè pour retomber à zéro. On obtient donc, non pas un courant continu-d'intensité constante - mais un courant pulsé unidirectionnel. Pour obtenir un courant continu, on relie des bobines comprenant un grand nombre de spires à des commutateurs composés d'un grand nombre de segments.

Courant continu, direct current (D.C.), continuous current, constant current. Courant unidirectionnel (-dont le sens ne change pas) d'intensité constante. Un courant unidirectionnel dont l'intensité varie est un courant pulsé (on trouve aussi courant pulsant, pulsatif, redressé et ondule). Comme un courant pulsé est toujours unidirectionnel, l'expression courant pulsé suffit. Eviter d'employer courant continu pulsé. 


\section{A.C. Generator}

Génératrice à courant alternatif ou Alternateur

Génératrice dont la tension de sortie est alternative

\section{Alternator}

V. A.C. Generator

\section{Armature}

Armature

Partie rotative d'un générateur usuel. Elle comprend la bobine et le commutateur.

Armature assembly

Armature, Induit

Toutes les pièces tournantes du générateur:

1. armature shaft $=$ arbre ou axe d'induit

2. armature core $=$ noyau d'induit

3. armature winding = enroulement d'induit

4. commutator $=$ commutateur.

Armature coil

Bobine d'armature

Enroulement de fil conducteur composé d'un grand nombre de spires et dont les deux extrémités sont reliées aux lames du commutateur.

Armature core

Noyau d'induit

Pièce ferro-magnétique, en acier ou en fer doux, en forme de tambour ou de cylindre sur laquelle est monté l'enroulement d'induit. Il permet de renforcer l'auto-inductance de l'enroulement, c. à d. la propriété d'induire une f.c.é.m. dans ses propres spires.

Armature reaction

Réaction d'induit

Réaction magnétique de l'induit qui déforme le champ magnétique principal engendré par l'inducteur et fait dévier le plan neutre et la ligne de calage des balais.

Armature shaft

Arbre d'induit

Axe métallique portant toutes les pièces qui composent l'armature ou induit. II tourne dans les coussinets (bearings) des cloches qu'on appelle aussi flasques ou paliers (frames).

Brush

Balai

Pièce conductrice de métal ou de graphite en contact avec les bagues collectrices ou le commutateur de la génératrice. Les deux balais transmettent la tension induite au circuit d'utilisation.

Brush gear

Porte-balais 
Gaine métallique isolée, du point de vue électrique, du flasque avant. Ils contiennent les balais et leurs connexions.

\section{Commutator}

Commutateur, collecteur

Cylindre formé par deux lames conductrices séparées par un isolant et reliées aux bornes de la bobine. Il sert à transformer le courant alternatif engendré par la génératrice en courant unidirectionnel.

Compensation windings

Bobines de compensation

Petits enroulements montés en série avec l'induit et logés dans des encoches des pièces polaires principales. Ils permettent d'annuler la réaction d'induit en créant un champ magnétique qui s'oppose à celui de l'induit.

Compound generator

Génératrice compound, Génératrice à excitation composée

Génératrice possédant un inducteur série et un inducteur shunt reliés à la sortie de la génératrice et formant un circuit série-parallèle. La compensation mutuelle des deux champs magnétiques rend la tension de sortie presque constante, quelle que soit l'intensité du courant débité.

\section{D.C. Generator}

Génératrice à courant continu ou Dynamo

Génératrice dont la tension de sortie est continue.

Drum-wound armature

Induit en tambour

Induit en cylindre formé de minces plaques d'acier dont l'assemblage présente des encoches dans lesquelles est insérée la bobine de telle sorte que les parties de chaque spire passent devant des pôles opposés.

\section{Dynamo}

Dynamo

Génératrice qui fournit du courant continu en redressant le courant alternatif obtenu dans l'induit au moyen d'un collecteur.

\section{Eddy current}

Courant de Foucault

Courant inutilisable induit dans les noyaux d'induit des génératrices. On le réduit par l'emploi de noyaux feuilletés (laminated), composés de minces plaques de métal isolées les unes des autres, au lieu de noyaux solides.

\section{Electromagnet}

Electroaimant

Aimant composé d'une bobine enroulé sur un noyau d'acier ou de fer doux. Le noyau devient magnétisé lorsque le courant circule dans la bobine et perd son magnétisme lorsque le courant est interrompu. Les électroaimants servent à engendrer le champ magnétique de la plupart des génératrices. 
Electromotive force ( $E M F$ )

Force électromotrice

Energie électrique ou force motrice capable de provoquer un courant. Elle apparaît dans l'armature lorsque celle-ci coupe des lignes de force du champ magnétique de la génératrice.

$E M F$

V. Electromotive force

Excitation current

Courant d'excitation

Courant continu qui traverse les bobines d'excitation pour produire le champ magnétique constant de la génératrice.

\section{Exciter}

Excitatrice

Génératrice à c.c. qui fournit le courant continu d'excitation chargé d'engendrer le champ magnétique d'une génératrice à excitation séparée.

Field coils

Bobines de champ

Électroaimants qui servent à produire le champ magnétique d'une génératrice.

Field frame

Carcasse, culasse

Partie centrale du bâti de la génératrice. Elle maintient les bobines de champ et sert à refermer le champ magnétique entre les pièces polaires.

Field winding

Enroulement de champ

Ensemble des bobines de champ.

Flat-compounded generator

Génératrice composée à plat, Génératrice compound à caractéristique horizontale

Génératrice compound dont les bobines d'excitation assurent une tension de sortie constante pour toutes les valeurs de charge comprises dans sa gamme.

\section{Front frame}

Flasque avant, Palier avant

Partie avant du bâti de la génératrice. Il maintient l'équipement de balais.

\section{Generator}

Génératrice

Appareil qui convertit l'énergie mécanique en énergie électrique au moyen d’un enroulement de fil conducteur tournant dans un champ magnétique. 
Heteropolar alternator

Alternateur hétéropolaire, Alternateur à flux alterné

Alternateur multipolaire dont les pôles nord et sud se succèdent alternativement sur le rotor. Le flux magnétique change ainsi de sens à chaque passage d'un pôle devant les bobines d'armature.

Homopolar alternator

Alternateur homopolaire, Alternateur à flux ondulé

Alternateur dont les pôles nord et les pôles sud sont respectivement situés d'un même côté de l'inducteur. Le flux magnétique varie autour d'une valeur moyenne dans l'induit.

Housing

Bâti

Charpente renfermant, soutenant et protégeant l'ensemble des pièces de la génératrice. Il comprend la carcasse et les flasques avant et arrière.

\section{Interlaced winding}

Enroulement imbriqué

Type d'enroulement de l'induit en tambour, dont les connexions sont imbriquées les unes dans les autres. I1 permet d'obtenir des courants puissants au moyen d'un grand nombre de pôles et d'un nombre correspondant de balais.

\section{Interpoles}

Pôles de commutation

Petits pôles auxiliaires placés entre les pièces polaires principales en série avec l'induit. Leur champ magnétique, opposé à celui de la bobine d'induit, annule la réaction d'induit.

\section{Lap winding}

V. Interlaced winding

\section{Left hand rule (for generators)}

Règle de la main gauche (pour les génératrices)

Méthode permettant de déterminer la f.é.m. induite dans un conducteur qui traverse un champ magnétique. Lorsque le pouce, l'index et le majeur de la main gauche forment entre eux des angles droits - l'index indiquant la direction du champ magnétique, et le pouce celle du mouvement du conducteur - le majeur indique le sens de la f.é.m. induite, c'est-à-dire la direction du courant qui en résulte.

\section{Loop}

Boucle, Tour, ou Spire (de bobine)

Conducteur unique formant un circuit fermé dont la rotation dans un champ magnétique induit une force électromotrice. La partie tournante - ou armature d'une génératrice comprend un grand nombre de spires.

\section{Magnetic field}

Champ magnétique

Espace compris entre les pôles de l'aimant permanent ou de l'électroaimant. La 
rotation d'une bobine de fil conducteur dans cet espace induit une force électromotrice dans la bobine.

Magneto (= Magnetoelectric generator)

Magneto (= Génératrice, magnets-électrique)

Petite génératrice à c.c. utilisant un ou plusieurs aimants permanents

Multipole alternator

Alternateur multipolaire

Alternateur comprenant deux ou plusieurs paires de pièces polaires (jusqu’à cinquante).

\section{Neutral plane}

Plan neutre, Plan de zéro

Position des lames du commutateur de la génératrice à courant continu lorsque la tension induite est égale à zéro. Chacune des lames est alors en contact avec les deux balais.

\section{Permanent magnet}

Airnant permanent

Pièce de métal qui conserve indéfiniment ses propriétés magnétiques. Ex.: l'acier dur et l'alnico (alliage d'aluminium, de fer, de nickel et de cobalt).

Pigtail

Queue de cochon

Fil électrique guipé (braided) flexible qui relie les balais aux porte-balais:

Pole pieces

Pièces polaires, Pôles inducteurs

Pièces magnétiques sur lesquelles sont enroulées les bobines de champ. L'espace compris entre les pièces polaires est occupé par l'armature.

Primary motor

Moteur primaire

Machine qui fournit à une génératrice l'ẻnergie nécessaire pour faire tourner le rotor. Ex.: machine à vapeur, turbine, moteur électrique, moteur à essence, etc.

\section{Rear frame}

Flasque arrière, Pallier arrière

Partie arrière du bâti de la génératrice. Il contient le support de l'induit.

\section{Ring-wound armature}

Induit en anneau

Induit comprenant plusieurs enroulements séparés par des intervalles égaux et montés sur un cylindre creux.

\section{Ripple}

Ondulation

Variation de la tension unidirectionnelle fournie par le commutateur de la génératrice 
élémentaire à courant continu. On la supprime en reliant plusieurs boucles à plusieurs lames de commutateur à raison d'une boucle pour deux lames (une lame pour chacune des deux extrémités de chaque boucle).

Rotating-armature alternator

Alternateur à induit tournant

Génératrice à courant alternatif dont l'induit tourne dans un champ magnétique fixe.

Rotating field alternator

Alternateur à inducteur tournant

Génératrice à c.a. dont l'enroulement d'induit est fixe et dont l'enroulement d'excitation est rotatif.

Rotor

Rotor

Partie rotative d'une génératrice à c.a. ou c.c., sur laquelle se trouvent l'induit, dans le cas des génératrices à c.c., et les bobines d'excitation (inducteurs) dans le cas des génératrices à c.a.

\section{Salient-pole rotor}

Rotor à pôles saillants

Rotor des alternateurs à petite vitesse (1200 tours max. par min.). Il comprend plusieurs pièces polaires bobinées séparément et vissées à la carcasse du rotor. Les extrémités des enroulements d'excitation sont reliées aux bagues collectrices montées sur l'axe du rotor.

\section{Self-excited generator}

Génératrice à auto-excitation

Génératrice à c.c. dont le champ magnétique est engendré par un courant d'excitation provenant de son propre débit.

Separately excited generator

Génératrice à excitation séparée

Génératrice à c.c. dont le champ magnétique est engendré par un courant d'excitation provenant d'une source extérieure, qui peut être une batterie ou une autre génératrice à c.c., appelée excitatrice.

\section{Series field-winding}

Inducteur-série

Inducteur formé par les bobines d'induction de la génératrice-série. Il est caractérisé par un nombre de spires relativement faible.

Series generator

Génératrice série

Génératrice à c.c. à auto-excitation dont les bobines d'excitation et le circuit extérieur sont en série. Ainsi, le même courant traverse l'induit, les bobines d'excitation formant l'inducteur, et le circuit extérieur ou la résistance de charge. 


\section{Shunt field-winding}

Inducteur shunt

Inducteur formé par les bobines d'induction de la génératrice shunt. Il est caractérisé par un grand nombre de spires en fil fin.

\section{Shunt generator}

Génératrice shunt

Génératrice à c.c. à auto-excitation dont les bobines d'excitation sont montées en shunt (ou en parallèle) avec l'induit. Ainsi, les bobines d'excitation ne sont traversées que par une petite partie du courant de l'induit, dont la plus grande partie traverse la résistance de charge.

\section{Sine wave}

Onde sinusoïdale

Forme de l'onde de tension produite par la génératrice à courant alternatif. Cette onde a la forme d'une courbe qui, partant de zéro, traverse toutes les valeurs de tension positives, revient à zéro, traverse toutes les valeurs négatives et revient à zéro.

\section{Single-phase alternator}

Alternateur monophasé

Alternateur dont l'induit présente des bobines reliées en série additive. Il fournit par conséquent une tension de sortie unique, d'amplitude égale à la somme des tensions induites dans chacune des bobines.

\section{Smooth-pole rotor}

Rotor à pôles lisses

Rotor des alternateurs à grande vitesse. Il présente des rainures pour loger solidement les conducteurs rectilignes de l'inducteur.

\section{Stationary-armature alternator}

V. Rotating field alternator

\section{Stator}

Stator

Partie fixe d'une génératrice à c.c. ou à c.a., sur laquelle se trouvent les bobines d'excitation (inducteurs) dans le cas des génératrices à c.c., et l'induit dans le cas des génératrices à c.a.

\section{Three-phase alternator}

Alternateur triphasé

Alternateur dont l'induit fixe consiste en trois enroulements monophasés dont les bobines sont espacées de façon que la tension induite dans chacun d'eux soit déphasée de 120 degrés par rapport aux tensions induites dans les deux autres enroulements.

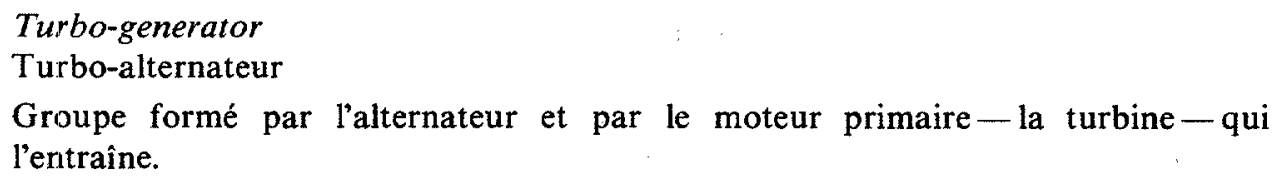


Two-phase alternator

Alternateur diphasé

Alternateur dont l'induit fixe consiste en deux enroulements monophasés, dont les bobines sont espacées de façon que la tension alternative induite dans l'un soit déphasée de 90 degrés par rapport à la tension alternative induite dans l'autre.

Two-pole generator

Génératrice bipolaire

Génératrice à deux bobines de champ.

Wave winding

Enroulement ondulé

Type d'enroulement de l'induit en tambour, dont chaque spire passe sous tous les pôles avant de revenir à son pôle de départ. Il permet d'obtenir de hautes tensions et n'utilise que deux balais.

Wye connection

Couplage en étoile

Type de montage permettant de relier la tension de sortie de l'alternateur à la charge extérieure. Il consiste à rassembler en un point commun appelé "point neutre» les conducteurs.

\section{Les problèmes d'équivalence en traduction technique}

Il arrive souvent que plusieurs termes anglo-américains n'ont qu'un seul équivalent français. La difficulté du choix se trouve alors écartée pour la traduction française, mais elle surgit lorsqu'on traduit en anglais. Voici quelques exemples courants tirés de la Traduction scientifique et technique, de Jean Maillot. Le terme échaufement correspond en anglais a heating ou à temperature rise, selon qu'il s'agit de l'aspect qualitatif ou de l'expression quantitative du phénomène. Fil se rend par wire ou thread, selon qu'il s'agit d'un conducteur électrique ou d'un fil textile. La gaine d'un câble fait l'objet d'une distinction pour les Américains, qui emploient jacket pour la gaine isolante et sheath pour la gaine métallique, mais les Britanniques emploient sheath dans les deux cas. Nous avons déjà parlé de la différence entre electric et electrical. À ce propos, nous constatons que le nom électrotechnique est plus employé que son équivalent electrotechnics auquel on préfère electrical engineering. Tension nominale se rend par rated-voltage pour un réseau de distribution ou un appareil, et par nominal voltage pour un réseau de transport. Souder se dit $t o$ solder ou to weld. Dans le premier cas, on joint les pièces de métal au moyen d'un alliage fondu sans les chauffer jusqu'au point de fusion. Dans le second, on les martèle ou on les comprime après les avoir assouplies en les chauffant.

Il arrive également qu'un terme anglo-américain présente plusieurs traductions suivant le cas. Manufacturer se rend ainsi par fabricant. On fabrique des lampes, écrit J. Maillot, mais on construit des machines. L'anglais conductivity se rend par conductibilité ou par conductivité, selon qu'il s'agit de la propriété de conduire le courant ou de la grandeur de cette propriété, pour laquelle l'anglais emploie 
parfois l'expression specific conductance. De même, à l'anglais insulation peuvent correspondre isolation: action d'isoler ou ensemble des isolants, isolement: ensemble des qualités acquises par un conducteur du fait de son isolation, enveloppe isolante et isolant.

$\mathrm{Au}$ français résistance correspondent en anglais deux termes, resistance, qui caractérise la grandeur exprimée en ohms, et resistor, qui désigne le composant, de métal ou de graphite, qui s'oppose au passage du courant. Pour éviter l'emploi du

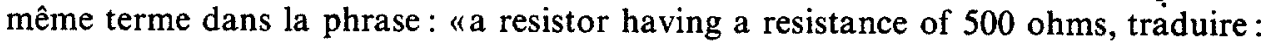
"une resistance dont la valeur est de 500 ohms".

En français comme en anglais, inductance désigne:

1) la propriété qui permet à une bobine d'induire, par l'effet d'une variation de courant à l'intérieur de la bobine, une force contre-électromotrice (f.c.é.m.) à ses bornes (phénomène d'auto-inductance ou inductance propre) ou dans une autre bobine (inductance mutuelle).

2) la grandeur, exprimée en henrys, qui mesure cette propriété.

3) le dispositif, appareil ou bobine, dont on utilise l'inductance.

Pour éviter toute confusion, on peut employer en français:

1) pour désigner la propriété: inductance propre ou auto-inductance, et inductance mutuelle.

2) pour désigner la grandeur: coefficient d'induction, d'induction propre, d'auto-induction et d'induction mutuelle. Éviter en français self et self-inductance.

3) pour désigner l'objet: bobine d'inductance.

en anglais :

1) Inductance, self inductance et mutual inductance.

2) Inductance.

3) Coil et inductor ${ }^{1}$.

L'anglais capacitance correspond au français capacité, charge électrique d'un condensateur. Mais le mot capacité est parfois employé pour désigner le condensateur lui-même. Le français capacitance est remplacé avantageusement par réactance capacitive, qui se dit en anglais capacitive reactance, pour lequel le dictionnaire de Piraux donne également un curieux condensance. Le français condensateur correspond à capacitor (E.U. et Canada) et à condenser (G.B.).

Ces quelques remarques montrent que la traduction technique ne saurait être considérée comme la substitution automatique de termes équivalents, car on ne rencontre guère d'équivalences parfaites. Cette complexité se trouve renforcée par les divergences croissantes entre l'anglais technique du Royaume-Uni et celui de l'Amérique du Nord. Nous étudierons dans un prochain numéro cet aspect du problème.

ARMAND A DLERBLUM

1. Inductor n'est pas un inducteur, qui dit field magnet ou field winding, suivant le cas, ou plus généralement field system. 